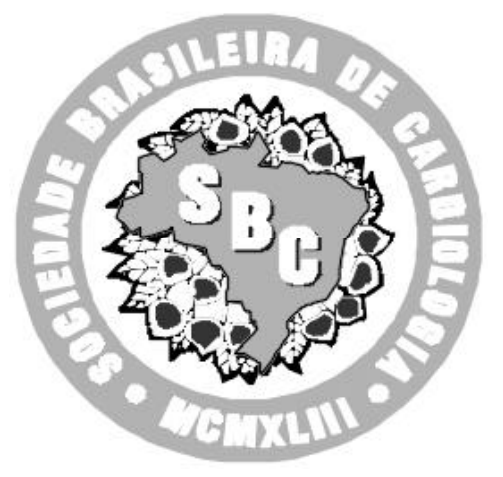

\title{
Diretriz de Indicações do \\ Ultra-Som Intracoronariano \\ na Prática Clínica
}




\title{
Diretriz de Indicações do Ultra-Som Intracoronariano na Prática Clínica
}

\author{
Coordenação: \\ Jorge Ilha Guimarães
}

Editor:

Alexandre Abizaid

Membros:

Constantino Costantine

Luis Alberto Mattos
Adriano Caixeta
Newton Stadler
Ibraim Pinto
Roselei Graebin
Gilvan Dourado
Claudia Alves
Andre Labrunie
Marcelo Freitas
Jamil Abdalla Saad

Jamil Abdalla Saad 


\section{I - Introdução}

Durante a última década, reconheceu-se que a angiografia coronariana não é o único método disponível para a visualização do interior das artérias coronarianas.

As primeiras imagens realizadas em vasos humanos foram gravadas por Yock e Linker, em 1988, e, no ano seguinte, foram feitas as primeiras observações por imagem da rede coronariana ${ }^{1,2}$. No final da década de 80 , vários grupos começaram a trabalhar no desenvolvimento de diferentes sistemas de cateteres, com o objetivo de obter imagens dos ateromas e, dessa forma, auxiliar as intervenções coronarianas percutâneas ${ }^{3}$. Em 1991, Yock e cols. publicaram trabalho pioneiro, em que utilizaram o ultra-som intracoronariano para visualizar, detalhadamente, o interior dos vasos e otimizar as técnicas de terapêutica invasiva, obtendo resultados animadores, principalmente quando foi utilizada a combinação de imagem com a intervenção ${ }^{4}$.

O ultra-som intracoronariano é uma técnica tomográfica que permite o estudo in vivo da parede vascular normal, dos componentes da placa aterosclerótica e das características quantitativas e qualitativas envolvidas no ateroma ${ }^{1,5}$. No campo da pesquisa, o ultra-som intracoronariano trouxe contribuições incontestes para o melhor conhecimento da doença aterosclerótica e do fenômeno da reestenose após a realização de intervenção coronariana percutânea ${ }^{6}$.

O objetivo desta diretriz é revisar, de maneira crítica, as definições e indicações do ultra-som intracoronariano na prática clínica, como método auxiliar no diagnóstico da doença arterial coronariana ou adjuvante à realização de um procedimento de revascularização miocárdica percutânea. Para tanto, foi empregada a classificação das evidências, de acordo com proposta do American College of Cardiology/ American Heart Association:

Classe I: condições em que existem evidências e/ou concordância geral de que o procedimento ou tratamento é benéfico, útil e efetivo.

Classe II: condições em que existem evidências conflitantes e/ou divergências nas opiniões sobre a utilidade e eficácia do procedimento ou tratamento.

Classe IIa: condições em que o peso das evidências e opiniões favorece a utilidade e eficácia do procedimento ou tratamento.

Classe IIb: condições em que a utilidade e eficácia do procedimento ou tratamento não estão muito bem estabelecidas por evidências e opiniões.

Classe III: condições em que existem evidências e/ou concordância geral de que o procedimento ou tratamento não é benéfico ou útil, podendo até ser deletério.

Nível de evidência $\mathbf{A}$ : dados oriundos de múltiplos ensaios clínicos randomizados

Nível de evidência $B$ : dados oriundos de um único ensaio clínico randomizado ou estudos não randomizados

Nível de evidência C: opiniões consensuais de especialistas no assunto.

\section{II -Considerações Técnicas}

O ultra-som intracoronarianoé um método de imagem de caráter invasivo, realizado em ambiente hospitalar, associado à metodologia utilizada para a realização de uma intervenção coronariana percutânea. A alta hospitalar pode ser efetivada após hemostasia da via de acesso e repouso de, no mínimo, 6h. O repouso poderá ser mais prolongado, na dependência do calibre do introdutor utilizado como via de acesso, em caso de punção femoral.

A) Via de acesso arterial - Pode ser utilizada tanto a punção femoral ou dissecção da artéria braquial, sendo o calibre mínimo necessário para a realização do exame igual ou maior que 6 French.

B) Material utilizado -É necessária a utilização de um cateter-guia, comum à prática da intervenção coronariana percutânea, com calibre igual ou superior a 6 French $(F)$ e luz interna superior a $0,64 \mathrm{~mm}$. Um fio-guia de 0,014 polegadas também é necessário, de preferência com um suporte reforçado. Os cateteres de ultra-som medem de 2,6 a 3,5 F.

C) Cateter de ultra-som - Existem dois tipos básicos de cateter: o sólido e o mecânico. Os dois tipos podem gerar imagem de 360 graus pelo eixo longitudinal. Os transdutores estão localizados na extremidade do cateter e apresentam, na grande maioria dos casos, frequiência de 30 megahertz. Os cateteres sólidos atuais têm 1,0 mm de diâmetro, ou seja, 3,2F, e são compatíveis com cateter guia de, no mínimo, 6F. Nesse cateter há diversos (64) transdutores posicionados radialmente à sua ponta. Esses transdutores são ativados por sequiência eletrônica. Uma vantagem do dispositivo sólido é que o sinal emitido pode ser controlado eletronicamente para obter, por exemplo, cortes em diferentes profundidades. O cateter sólido, no entanto, pode exibir um artefato ao seu redor (chamado de ring-down), produzido por oscilações acústicas dos transdutores, que prejudicam o campo de visão, acarretando a visualização de um tamanho de cateter maior que o normal. O cateter mecânico, por sua vez, tem perfil menor que o sólido, porém podem ocorrer artefatos, mais frequientemente.

D) Técnica de imageamento - Todas as avaliações ultra-sonográficas são realizadas após a administração de nitroglicerina intracoronariana, na dose de $0,2 \mathrm{mg}$ e heparina endovenosa $100 \mathrm{U} / \mathrm{kg}$. Para cada seqüência de imagens, avança-se o cateter de ultra-som, aproximadamente $10 \mathrm{~mm}$, distalmente à lesão-alvo; a partir desse ponto, inicia-se a aquisição ininterrupta de imagens, até a junção aorto-ostial. Ramos visualizados pela angiografia ou pelo ultra-som são marcadores importantes, que facilitam a interpretação e comparação com exames subseqüentes. Para obter a sequiência de imagens, o transdutor de ultra-som é tracionado por um equipamento automático, à velocidade de $0,5 \mathrm{~mm} / \mathrm{s}$. Os exames de ultra-som são gravados e arquivados em fitas de vídeo de alta resolução para análise em laboratório especializado. Alguns grupos utilizam a reconstrução tridimensional da imagem. Esse processo envolve o corte de várias imagens bidimensionais ao longo de uma 
distância conhecida. $\mathrm{O}$ vaso pode ser exibido em diversas posições no espaço, inclusive longitudinalmente, facilitando apreciações morfológicas detalhadas.

\section{III - Interpretação do ultra-som intracoronariano}

Na maioria dos vasos (na vigência de algum grau de ateromatose), o ultra-som permite visualizar as três camadas arteriais: íntima, média e adventícia. No entanto, em artérias totalmente normais, a visualização torna-se mais restrita, pois as camadas íntima e média são microscópicas e difíceis de serem visualizadas. Nelas, duas interfaces são observadas: na transição entre o sangue e a íntima e na transição entre a média e a adventícia, essa chamada de membrana elástica externa (fig. 1). Em 30 a 50\% dos casos em que as artérias coronarianas são normais, a camada íntima é fina e reflete pouco ultra-som, portanto não pode ser visualizada como uma camada separada ${ }^{7}$. A túnica média tem pouco conteúdo de colágeno e é constituída, basicamente, por tecido muscular, sendo tipicamente ecoluscente ao ultrasom. A média aparece como camada escura em relação à íntima e à adventícia e seu grau de espessamento é proporcional ao grau de aterosclerose. A adventícia tem alto conteúdo de colágeno, portanto é uma camada ecorrefletora ao ultra-som. O seu limite interno, isto é, a transição entre a camada média e a adventícia, é bem visível ao ultra-som, sendo denominada membrana elástica externa. Entretanto, os limites externos da adventícia são imprecisos ao ultrasom, pois os tecidos periadventiciais se confundem com os da adventícia, por apresentar em quantidades semelhantes de colágeno e elastina ${ }^{5,8-10}$.

A) Análise da imagem - O ultra-som tem capacidade de distinguir os quatro tipos morfológicos básicos de placa aterosclerótica, de acordo com a ecodensidade: lipídica, fibrótica, calcificada e mista. A camada adventícia, rica em colágeno e altamente ecorreflexível, é utilizada como referência. Além disso, vários estudos compararam os aspectos ultra-sonográficos das placas ateroscleróticas in vivo com achados histopatológicos post-mortem ${ }^{9,11}$, comprovando a fidelidade dos achados. O quadro I descreve os diferentes tipos de placa.

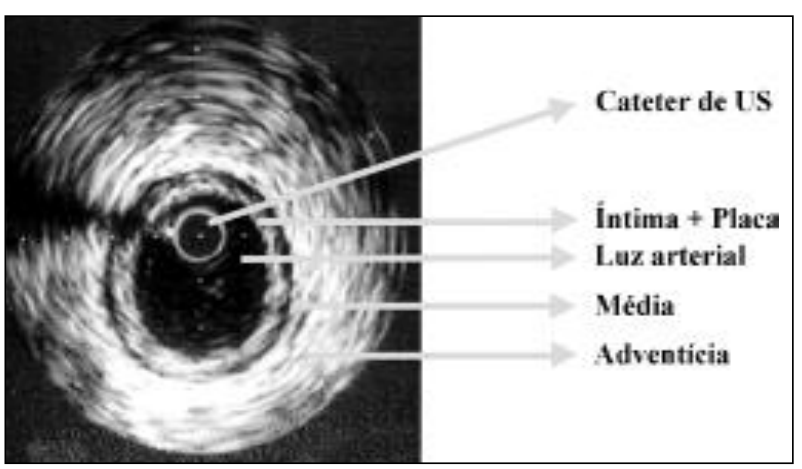

Fig. 1 - Imagem ultrasonográfica de secção transversa da artéria coronária evidenciando suas diferentes camadas: íntima, média e adventícia.
B) Avaliação qualitativa-O método permite diferenciar, com boa precisão, as principais alterações ocorridas em uma placa de ateroma. O quadro II descreve estes achados. Um achado frequiente é o remodelamento arterial (fig. 5). Este termo se refere às mudanças nas dimensões vasculares durante a progressão da aterosclerose. Como descrito em necropsia, nada mais é do que o aumento compensatório do vaso para acomodar uma grande quantidade de placa, na tentativa de preservar a luz arterial ${ }^{12}$. É também chamado de remodelamento adaptativo, que age "escondendo" porções de placa à avaliação pela angiografia coronariana. Estudos com ultra-som demonstraram haver dois tipos de remodelamento: positivo, que é descrito quando a área da membrana elástica externa, no local da lesão, aumenta em relação ao segmento de referência, durante o processo de desenvolvimento do ateroma; e negativo, em que a diminuição da membrana elástica externa contribui no processo de

\begin{tabular}{|c|c|}
\hline \multicolumn{2}{|c|}{$\begin{array}{l}\text { Quadro I - Descrição dos diversos tipos de placa encontrados pelo } \\
\text { ultra-som intracoronariano e utilizados na interpretação do exame. }\end{array}$} \\
\hline Tipo de placa & Característica \\
\hline Lipídica & \\
\hline & $\begin{array}{l}\text { Placa ecoluscente (a que } \\
\text { reflete ou brilha menos do } \\
\text { que a adventícia), heterogê- } \\
\text { nea, denotando a presença de } \\
\text { lagos gordurosos. }\end{array}$ \\
\hline Fibrótica & \\
\hline & $\begin{array}{l}\text { Placa que se mostra ecodensa } \\
\text { ao ultra-som (tanto ou mais } \\
\text { ecorreflexível que a camada } \\
\text { adventícia), porém sem } \\
\text { produzir sombra acústica, } \\
\text { como ocorre nas placas } \\
\text { calcificadas. Constitui a } \\
\text { maioria das lesões } \\
\text { ateroscleróticas. }\end{array}$ \\
\hline Calcificada & \\
\hline & $\begin{array}{l}\text { Placa definida pela presença } \\
\text { de cálcio, um refletor muito } \\
\text { forte de ultra-som, verdadeiro } \\
\text { escudo sobre todas as } \\
\text { estruturas vasculares que se } \\
\text { localizam nas camadas mais } \\
\text { profundas, produzindo } \\
\text { sombra acústica e, ocasional- } \\
\text { mente, reverberação. }\end{array}$ \\
\hline Mista & $\begin{array}{l}\text { Diagnosticada na presença de } \\
\text { mais de um dos três } \\
\text { componentes (lípides, fibrose } \\
\text { e cálcio) na mesma placa. }\end{array}$ \\
\hline
\end{tabular}




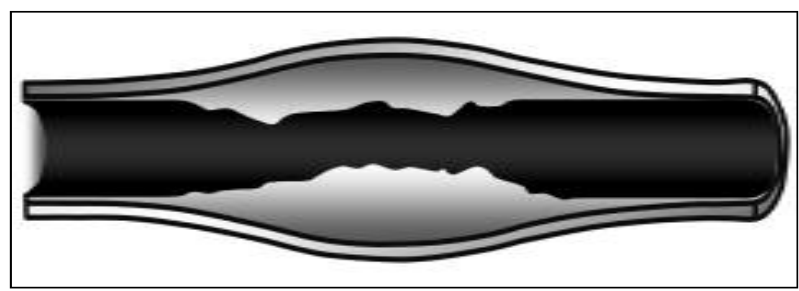

Fig. 5 - Representação gráfica de remodelamento positivo. Ocorre um processo de aumento da membrana elástica externa na tentativa de acomodar a placa aterosclerótica em crescimento.

estenose coronariana e que tem sido visto como responsável pela reestenose após intervenções coronarianas, que não utilizaram implante de stents $^{12-15}$.

C) Avaliação quantitativa - As medidas quantitativas do ultra-som são realizadas em três segmentos da artéria coronariana: nas referências proximal e distal do vaso e no local mais crítico da lesão coronariana. Os locais para as medidas das referências são selecionados até $10 \mathrm{~mm}$ proximal e distalmente aos respectivos bordos da lesão e devem exibir aparência a mais normal possível. A extensão da lesão é calculada por meio da tração automática do equipamento. Esse dispositivo traciona o transdutor de ultra-som à velocidade programada de $0,5 \mathrm{~mm}$ por segundo. A tração deve ser realizada do segmento distal para o proximal da lesãoalvo. As diversas fórmulas utilizadas e suas definições são:

Diâmetro de referência: resultado da média aritmética dos maiores diâmetros dos segmentos de referências distal e proximal; expresso em milímetros;

Área da luz da referência: resultado da média aritmética das áreas das luzes das referências proximal e distal, expressa em milímetros quadrados;

Área da membrana elástica externa (MEE): apesar de MEE ser uma camada microscópica, sabe-se que se situa entre as camadas média e adventícia, por isso, sua área é obtida a partir do delineamento da transição entre essas duas camadas, sendo expressa em milímetros quadrados;

Área mínima da luz (AML): a partir do delineamento da transição entre a luz e a camada íntima, tem-se a AML, expressa em milímetros quadrados;

Percentagem do vaso ocupada pela placa (\%Placa): calculada por uma fórmula $(\% \mathrm{Placa}=\mathrm{P}+\mathrm{M} / \mathrm{MEE})$.

\section{IV - Utilização de ultra-som intracoronariano no Brasil}

O exponencial crescimento da prática da intervenção coronariana percutânea no Brasil está documentado por meio do registro CENIC (Central Nacional de Intervenções Cardiovasculares), pertencente à SBHCI (Sociedade Brasileira de Hemodinâmica e Cardiologia Intervencionista) que, desde 1991, contabiliza os procedimentos percutâneos cardiovasculares realizados em nosso país. De 1997 até 2000, 133 hospitais brasileiros enviaram o relatório acerca de 41.821 pacientes submetidos à intervenção coronariana percutânea com implante de stent coronariano. Destes, 2.359 $(5,6 \%)$ realizaram o ultra-som intracoronariano associado ao procedimento de revascularização percutâneo (tab. I). Os pacientes que utilizaram o método obtiveram uma menor estenose residual (análise visual) e percentual reduzido de complicações imediatas maiores, analisadas até a alta hospitalar. Por meio da análise de regressão logística multivariável, os autores identificaram, como preditores independentes para ocorrência de desfechos adversos, a não realização do ultrasom, a idade avançada, a presença de trombo e a estenose residual após a realização do procedimento.

\begin{tabular}{|c|c|}
\hline \multicolumn{2}{|c|}{$\begin{array}{l}\text { Quadro II - Diversas alterações identificadas ao ultra-som } \\
\text { intracoronariano e suas características qualitativas. }\end{array}$} \\
\hline Alteração & Característica \\
\hline Placa instáve & $\begin{array}{l}\text { Placas hipoecóicas, ricas em } \\
\text { lipídeos e com uma capa } \\
\text { fibrosa fina. }\end{array}$ \\
\hline Rotura da pla & $\begin{array}{l}\text { Ulceração com rotura da capa } \\
\text { fibrótica, associada a fissuras } \\
\text { e erosões da sua superfície. } \\
\text { Pode ser feita injeção de } \\
\text { contraste para definir o ponto } \\
\text { de comunicação. }\end{array}$ \\
\hline Ulceração & $\begin{array}{l}\text { Caracterizada como uma } \\
\text { fissura iniciada no bordo da } \\
\text { luz arterial com a íntima, sem } \\
\text { causar aumento na membrana } \\
\text { elástica externa (comparada } \\
\text { ao segmento de referência } \\
\text { luminal). }\end{array}$ \\
\hline Trombo & $\begin{array}{l}\text { Massa lobulada ou } \\
\text { pedunculada ecoluscente, } \\
\text { que se projeta para a luz } \\
\text { arterial, associada à } \\
\text { movimentação ondulatória e } \\
\text { presença de espículas } \\
\text { sanguíneas, indicando } \\
\text { microcanais. A identificação } \\
\text { nem sempre é fácil, } \\
\text { constituindo uma limitação } \\
\text { deste método. }\end{array}$ \\
\hline Dissecção & $\begin{array}{l}\text { Analisada segundo a } \\
\text { profundidade, circunferência } \\
\text { e extensão comprometidas. }\end{array}$ \\
\hline
\end{tabular}




\begin{tabular}{|c|c|c|c|}
\hline \multicolumn{4}{|c|}{$\begin{array}{c}\text { Tabela I - Resultados do registro CENIC (Central Nacional de } \\
\text { Intervenções Cardiovasculares), acerca do implante de stent } \\
\text { coronariano, com ou sem a monitoração do ultra-som, de } 1997 \\
\text { até } 2000 \text {. }\end{array}$} \\
\hline Implante de stent & Com ultra-som & Sem ultra-som & $P$ \\
\hline Número de pacientes & $2,359(5,6 \%)$ & $39,462(94,4 \%)$ & - \\
\hline Idade média & $59,7 \pm 10,9$ anos & $60,7 \pm 11,1$ anos & $<0,001$ \\
\hline Sexo masculino & $70,4 \%$ & $69,9 \%$ & 0,585 \\
\hline Diabéticos & $16,5 \%$ & $17,1 \%$ & 0,488 \\
\hline Angina instável & $24,0 \%$ & $21,1 \%$ & 0,001 \\
\hline Trombo presente & $18,6 \%$ & $20,1 \%$ & 0,068 \\
\hline Calcificação & $31,4 \%$ & $19,0 \%$ & $<0,001$ \\
\hline Sucesso inicial & $98,9 \%$ & $98,6 \%$ & 0,213 \\
\hline$\%$ Estenose final & $8,3 \pm 10,8 \%$ & $10,6 \pm 11,6 \%$ & $<0,001$ \\
\hline Infarto do miocárdio & $0,6 \%$ & $1,0 \%$ & 0,066 \\
\hline Mortalidade & $0,9 \%$ & $1,8 \%$ & 0,001 \\
\hline
\end{tabular}

Portanto, o uso clínico do ultra-som permanece restrito, mas demonstra ser seguro e eficaz, como evidenciado pelos resultados do registro CENIC.

\section{V - Indicações clínicas do ultra-som intracoronariano}

As indicações podem variar, desde o diagnóstico, como identificador da presença de doença arterial coronariana, até o monitoramento de procedimentos de intervenção percutâneos.

A) Diagnóstico da doença arterial coronariana Embora a angiografia seja o método padrão para avaliar a doença arterial coronariana e para guiar intervenção percutânea, o mesmo exibe algumas limitações. A aterosclerose é uma doença da parede do vaso, e a angiografia estuda apenas sua luz, diagnosticando lesões obstrutivas pela comparação entre segmentos doentes e segmentos "supostamente" normais. Porém, muitas vezes, esses segmentos chamados normais, na verdade, podem exibir o fenômeno de ampliação do vaso, ocorrido durante o processo de aterosclerose, na tentativa de acomodar a placa (remodelamento positivo) ${ }^{16-19}$. Como o ultra-som permite análise tomográfica de todas as camadas arteriais, ele se torna um método mais acurado para análise das dimensões arteriais. Em um estudo, foram analisadas 884 lesões em vasos nativos, em segmentos de referência angiograficamente "normais", sendo demonstrado, por meio do ultra-som, que apenas 6,8\% desses segmentos eram realmente livres de doença ${ }^{14}$.

O método pode ser utilizado para esta finalidade, desde que exista correlação com os dados clínicos do paciente. A gravidade da doença arterial coronariana e a necessidade de realizar um procedimento de revascularização também devem ser substanciados pela presença de sinais de isquemia miocárdica em provas funcionais não invasivas (Classe IIa, nível de evidência B).

B) Avaliação da gravidade das estenoses coronarianas (tronco da coronária esquerda) - Três fatores anatômicos comprometem a avaliação angiográfica do tronco da coronária esquerda (TCE): a dificuldade de se visualizar, de maneira precisa, o TCE por opacificação da cúspide aórtica, a presença de bifurcação ou trifurcação, que pode ocultar a parte distal do TCE e a pequena extensão do TCE. Não existe consenso na literatura em relação a parâmetros ultrasonográficos para se intervir ou não em lesões localizadas no TCE ${ }^{20,21}$. Porém, a percentagem de estenose $>50 \%$ (comparada com a área do lúmen de referência), o diâmetro mínimo da luz $<2 \mathrm{~mm}$ e/ou área mínima absoluta da luz arterial $<6 \mathrm{~mm}^{2}$ têm sido considerados critérios satisfatórios para indicação de intervenção cirúrgica ou percutânea ${ }^{22}$ (Classe IIa, nível de evidência B).

C) Avaliação da gravidade de estenoses coronarianas (lesão moderada) - Inúmeros pacientes exibem a presença de estenoses consideradas de grau intermediário ou moderadas, por meio da realização da angiografia coronariana $(>50-<70 \%)$. Esses pacientes podem ou não apresentar sintomas anginosos, ou, até mesmo, provas funcionais de detecção de isquemia miocárdica positivas ou duvidosas. Portanto, muitos constituem uma indefinição para a indicação de um procedimento de revascularização. A análise de uma série unicêntrica e consecutiva de casos com essas características demonstrou que pacientes com áreas luminais inferiores a $4,0 \mathrm{~mm}^{2}$ apresentaram uma evolução clínica significativamente menos favorável ao final de um ano de seguimento, relacionada à maior ocorrência de desfechos adversos somados (necessidade de revascularização, infarto do miocárdio e óbito), totalizando cerca de duas vezes mais do que no caso de pacientes submetidos a uma intervenção coronariana percutânea ou permaneceram em tratamento clínico ${ }^{23}$. Assim, o método pode constituir-se em importante auxílio na definição desses casos. É importante salientar que este parâmetro apresenta uma correlação mais fiel, quando o diâmetro de referência do vaso analisado é igual ou superior a 3,0 mm (Classe IIa, nível de evidência B).

D) Detecção de placas instáveis - Síndromes coronarianas agudas, freqüentemente, se desenvolvem em território com pouca doença e não em vasos com estenose de maior gravidade ${ }^{16,24,25}$. Uma aplicação potencial do ultrasom intracoronariano tem sido a identificação do ateroma com risco de rotura ${ }^{26-32}$. A histologia das placas instáveis, usualmente, revela um ateroma rico em lipídios com uma capa fibrótica fina ${ }^{6,9}$. O ultra-som também tem demonstrado a associação das placas ecoluscentes, ricas em lipídios, geralmente com a presença de lagos lipídicos e capa fibrótica delgada com síndromes coronarianas agudas ${ }^{33-36}$. Recentemente, um outro dado ultra-sonográfico tem sido associado às síndromes instáveis: a presença do remodelamento arterial positivo ${ }^{12-14}$. Além disso, a presença de trombos intralûminas, fissuras ou rotura na placa são considerados como marcadores da síndrome coronariana aguda ${ }^{37-41}$.

Comparados a pacientes com angina estável, os pacientes com angina instável apresentaram uma incidência maior de placas lipídicas (74\% vs. $41 \%$; p $<0.01$ ) e uma incidência menor de placas calcificadas e fibróticas ( $25 \%$ vs. $45 \%$; $p<0.01$ ), em análise de 65 pacientes $^{42}$. É interessante ressaltar que não houve correlação entre as características morfológicas das lesões encontradas no ultra-som e na angiografia coronariana, sendo que o ultra-som apresentou 
uma maior sensibilidade na identificação de lesões instáveis, quando comparado à angiografia coronariana ( $74 \%$ vs. $40 \%$, respectivamente). Rasheed e cols. estudaram 146 pacientes com diferentes quadros clínicos e concluíram que aqueles casos com angina em repouso e/ou angina pósinfarto apresentam um número expressivo de lesões lipídicas $(71 \%$ e $73 \%$, respectivamente), enquanto pacientes com angina estável e/ ou angina crescente apresentam uma incidência maior de placas fibróticas ou calcificadas $(69 \%$ e $53 \%$, respectivamente $)^{43}$. Outro estudo demonstrou que pacientes portadores de síndrome instável, quando comparados com o mesmo número de casos com angina estável, apresentam, ao ultra-som, camada mais fina circundando a íntima (77\% vs. $7 \%$ ) e placas mais ecoluscentes (72\% vs. $46 \%)^{44}$.

Na vigência de infarto agudo do miocárdio, também foi realizada a análise da placa aterosclerótica ${ }^{45-49}$. A detecção da presença de trombos nestes estudos foi alta, variando de $70 \%$ a $90 \%$, e, quando o ultra-som foi comparado à histologia, a taxa de acurácia foi de $80 \%{ }^{37}$. Em alguns estudos, demonstrou-se a presença de lesões excêntricas em torno de $80 \%$, porém a prevalência de fissuras ou dissecções ocorreu em menos de $30 \%$ dos pacientes, após o evento agudo $^{45,46}$. Abizaid e cols. estudaram 60 pacientes até $72 \mathrm{~h}$ após infarto do miocárdio e identificaram, na grande maioria dos pacientes, placas lipídicas, com apenas $10 \%$ de placas calcificadas. O remodelamento arterial positivo no local da lesão foi diagnosticado em $86 \%$ dos casos, sendo que $85 \%$ dos pacientes apresentaram evidência de trombos ao ultra-som, $90 \%$, presença de rotura de placa e associação de trombos com rotura foi observada em $98 \%$ dos $\operatorname{casos}^{49}$. A utilização de ultra-som nesses pacientes é interessante, pois fornece mais fundamento à realização de um procedimento de revascularização do miocárdio, além de poder se constituir em um método de seguimento, após a ocorrência de uma síndrome coronariana aguda (Classe IIa, nível de evidência B).

E) Ultra-som como método de monitoração de uma intervenção coronariana percutânea (stents) - O ultra-som é capaz de detectar alterações na luz do vaso, que, apesar de relativamente pequenas (em média de $0,5 \mathrm{~mm}$ ), apresentam impacto clínico significativo. Essas alterações poderiam determinar condutas distintas, seja durante o implante, elevando a pressão de impactação da endoprótese na parede do vaso, aumentando o calibre do balão ou, quando da realização da cinecoronariografia tardia, auxiliando na decisão de revascularizar o vaso previamente tratado ${ }^{50}$.

Em 1998, o estudo MUSIC (Multicenter Ultrasound Stenting in Coronaries Study) despertou o interesse para o fato de que o implante do stent guiado pelo ultra-som demonstrou taxas de reestenose menores $(8,3 \%)^{51}$. A seguir, outras investigações avaliaram, de forma comparativa e randomizada, qual o melhor método guia para o implante do stent coronariano, comparando o ultra-som intracoronariano com a angiografia coronariana quantitativa, as possíveis implicações clínicas relacionadas à ocorrência de reestenose intra-stent e a necessidade de realizar novos procedimentos de revascularização miocárdica ${ }^{52,53}$.

Os resultados de cinco estudos randomizados foram conflitantes (tab. II). Três estudos que realizaram uma nova cinecoronariografia tardia e, assim, de fato aferiram as taxas de reestenose intra-stent, não demonstraram diferença estatisticamente significante. Dois estudos relataram significativa redução na cifra de nova revascularização do vasoalvo, utilizando, contudo, método menos uniforme. O estudo CRUISE (Can Routine Ultrasound Influence Stent Expansion), que mostrou a superioridade do ultra-som, não realizou nova cinecoronariografia, e o SIPS (Strategy for Intracoronary Ultrasound-Guided PTCA and Stenting), relata similaridade nas taxas de reestenose angiográfica intra-stent aos seis meses (29\% versus 35\%, ultra-som e angiografia quantitativa; $\mathrm{p}=\mathrm{NS}$ ), porém informa significante redução na realização de novos procedimentos de revascularização do vaso-alvo em período mais prolongado, após dois anos de evolução clínica ( $17 \%$ versus $29 \%$, ultra-som e angiografia quantitativa; $\mathrm{p}=0,02)^{54,55}$. Por outro lado, no estudo OPTICUS (Optimization with Intracoronary Ultrasound to reduce stent restenosis), os pacientes que tiveram o implante do stent monitorado por meio do ultra-som intracoronariano necessitaram de maior quantidade de meio de contraste e o dobro do tempo de radioscopia, elevando o tempo total do procedimento (em quase 30min). Essas diferenças foram estatisticamente significantes e associadas à constatação de nenhum benefício tardio, em relação à redução das cifras de reestenose clínica e angiográfica ${ }^{56}$. É importante salientar que esses estudos analisaram estenoses de comprimento relativamente curto, geralmente inferior a $20 \mathrm{~mm}$.

\begin{tabular}{|lcc|}
\hline \multicolumn{4}{|c|}{ Tabela II - Sumário dos resultados observados nos estudos randomizados, que compararam o ultra-som e a angiografia coronariana quantitativa, } \\
como guia para o implante de stent coronariano.
\end{tabular}


No momento atual, não existe uma recomendação formal para utilização do ultra-som intracoronariano de forma sistemática, como método auxiliar do implante dos stents. Seu uso pode otimizar os resultados imediatos, oferecendo um ganho luminal adicional imediato maior, mas a extensão desse benefício à redução das taxas de reestenose ainda é controversa. (Classe IIb, nível de evidência A).

F) Ultra-som como método de monitoração de uma intervenção coronariana percutânea (stent no tratamento de estenoses longas) - Estudo randomizado recente, TULIP (Thrombocyte active evaluation and effects of Ultrasound guidance in Long Intracoronary stent Placement) avaliou a possibilidade de benefício tardio, representado por menores taxas de reestenose intra-stent tardias e suas conseqüências clínicas, em portadores de estenose-alvo de comprimento igual ou superior a $20 \mathrm{~mm}$, submetidos ao implante de stents $^{57}$.

Cento e quarenta e quatro portadores de estenoses longas (29 $\pm 10 \mathrm{~mm}$ versus $27 \pm 9 \mathrm{~mm}$, respectivamente; $\mathrm{p}=\mathrm{NS}$ ) foram randomizados para o monitoramento com ultra-som ou angiografia coronariana quantitativa, em vasos com diâmetro igual ou superior a 3,0 $\mathrm{mm}$. Uma nova angiografia coronariana foi realizada após 6 meses do procedimento e a evolução clínica completada até os 12 meses. Os stents utilizados foram múltiplos $(1,4 \pm 0,6$ versus $1,1 \pm 0,4$, ultra-som e angiografia; $\mathrm{p}<0,001)$ e longos $(42 \pm 11 \mathrm{~mm}$ versus $35 \pm 11 \mathrm{~mm}$, ultra-som e angiografia; $\mathrm{p}=0,001)$. Aqueles pacientes que implantaram stents longos, auxiliados por meio do ultra-som, obtiveram uma luz do vaso-alvo maior, imediatamente após o procedimento $(3,01 \pm 0,4 \mathrm{~mm}$ versus $2,80 \pm 0,3 \mathrm{~mm} ; \mathrm{p}=0,008)$, assim como na avaliação angiográfica tardia $(1,82 \pm 0,5 \mathrm{~mm}$ versus $1,51 \pm 0,7 \mathrm{~mm} ; \mathrm{p}=0,042)$, sendo a reestenose intra-stent significativamente menor $(23 \%$ versus 46\%; $\mathrm{p}=0,008)$. Ao final de um ano de evolução, o grupo de pacientes que realizou o procedimento monitorado pelo ultra-som necessitou de um número menor de novas revascularizações do vaso-alvo (10\% versus $23 \%$; $\mathrm{p}=0,018)$, tendo evoluído com número menor de eventos combinados cumulativos maiores ( $12 \%$ versus $27 \% ; \mathrm{p}=0,026)$.

Nesses casos, o auxílio do ultra-som intracoronariano parece ser mais significativo que em relação a estenoses mais focais, havendo a possibilidade de sua utilização ser benéfica, relacionada à obtenção de uma melhor consolidação tardia da intervenção coronariana percutânea com o implante de stents (Classe IIa, nível de evidência B).

G) Ultra-som no tratamento da reestenose intrastent: Na reestenose intra-stent, o ultra-som pode ser utilizado para identificar o mecanismo da reestenose e, dessa forma, guiar a melhor opção terapêutica. Esses mecanismos incluem má aposição, má expansão ou falha em cobrir toda a lesão alvo ${ }^{58}$. Castagna e cols avaliaram 1090 pacientes com reestenose intra-stent e encontraram algum achado morfológico que contribuiu para a reestenose em $4.5 \%$ dos pacientes ${ }^{59}$. Esses achados incluíram falha em cobrir toda a lesão (principalmente estenoses aorto-ostiais), fratura ou deslocamento do stent durante o implante. $20 \%$ dos casos apre- sentaram área de seção transversal < $80 \%$ da média da área luminal de referência e uma área mínima do stent $\mathrm{de}<5 \mathrm{~mm}^{2}$, evidenciando, portanto, má expansão da endoprótese. Além disso, o ultra-som permite determinar se a hiperplasia intimal ocorreu apenas intra-stent ou incluiu o segmento de referência adjacente, fato esse que orienta o implante de stent adicional. (Classe II, Nível de evidência, B)

Outra aplicação do ultra-som se faz na monitorização da braquiterapia para o tratamento da reestenose intrastent, em que, além de avaliar o resultado da intervenção percutânea antes da aplicação da radiação, é fundamental para a prescrição da dose do radiofármaco a ser utilizada, com base no diâmetro do vaso. (Classe I, Nível de evidência B).

H) Ultra-som na monitoração do implante de stents eluídos em fármacos - Uma nova fase da cardiologia intervencionista foi iniciada, em 1999, com a apresentação dos primeiros resultados obtidos após o implante de stents coronarianos eluídos com fármacos antiproliferativos, que auxiliam na redução das taxas de reestenose tardias ${ }^{60-65}$. Desde então, essa nova tecnologia vem sendo, progressivamente, incorporada à prática clínica, visto a evidência dos bons resultados imediatos e tardios, superiores aos observados, até o momento, com os stents convencionais. O ultra-som auxiliou na fundamentação desta nova técnica, comprovando a segurança e eficácia tardia. O método demonstrou que estes novos stents não promovem anormalidades tardias significativas na parede do vaso tratado (formação de aneurismas), resultando uma melhor consolidação do processo de cicatrização tardio, com evidência de mínima hiperplasia mio-intimal obstrutiva. As taxas de reestenose foram reduzidas, quando comparadas com aquelas observadas após o implante de stents convencionais (>50\%). Houve benefícios clínicos associados, representados por menor necessidade de repetição dos procedimentos de revascularização do miocárdio. A utilização do ultra-som na análise tardia dos resultados desta nova tecnologia é importante, visto que ainda existem inúmeras pesquisas em andamento, relacionadas a diferentes fármacos e endopróteses em fase de experimentação (Classe IIb, nível de evidência A).

\section{VI - Considerações finais}

O ultra-som intracoronariano promoveu um avanço nas técnicas de imagem intracoronarianas, auxiliando no estudo da doença arterial coronariana, sua evolução e, principalmente, na análise da ocorrência de eventos associados à realização de uma intervenção coronariana percutânea. $\mathrm{O}$ método foi importante para a melhoria dos resultados imediatos e tardios, observados após o implante de stents coronarianos, contribuindo para a segurança, difusão e equalização desta técnica. Por outro lado, o ultra-som eleva mais o custo do procedimento, gasto adicional de meio de contraste e um tempo mais prolongado para a sua efetivação, porém sem provocar, diretamente, a ocorrência de complicações maiores. Esses aspectos devem ser julgados quando da sua indicação, fundamentando sua solicitação. 


\section{Referências}

1. Yock PG, Linker DT. Intravascular ultrasound, looking below the surface of vascular disease. Circulation 1990;81:1715-8.

2. Yock PG, Linker DT, Angelsen BA. Two-dimensional intravascular ultrasound: technical development and initial clinical experience. J Am Soc Echocardiogr. 1989;2:296-304.

3. Gussenhoven EJ, Essed CE, Lancee CT, et al. Arterial wall characteristics determined by intravascular ultrasound imaging: an in vitro study. J Am Coll Cardiol. 1989;14:947-52.

4. Yock PG, Fitzgerald PJ, Sudhir K, et al. Intravascular ultrasound imaging for guidance of atherectomy and other plaque removal techniques. Int J Card Imaging. 1991;6:179-89.

5. Potkin BN, Bartorelli AL, Gessert JM, et al. Coronary artery imaging with intravascular high frequency ultrasound. Circulation 1990;81:1575-85.

6. Tobis JM, Mallery J, Mahon D, et al. intravascular ultrasound imaging of human coronary arteries in vivo. Analysis of tissue characterizations with comparison to in vitro histological specimens. Circulation 1991;83:913-26.

7. Nissen SE, Gurley JC, Grines CL, et al. Intravascular ultrasound assessment of lumen size and wall morphology in normal subjects and patients with coronary artery disease.Circulation 1991;84:1087-99.

8. St Goar FG, Pinto FJ, Alderman EL, et al. Intravascular ultrasound imaging of angiographically normal coronary arteries: an in vivo comparison with quantitative angiography. J Am Coll Cardiol. 1991;18:952-8.

9. Nishimura RA, Edwards WD, Warnes CA, et al. Intravascular ultrasound imaging: in vitro validation and pathologic correlation. J Am Coll Cardiol 1990; 16:145-54.

10. Fitzgerald PJ, St. Goar FG, Connolly AJ, et al. Intravascular ultrasound imaging of coronary arteries. Is three layers the norm? Circulation 1992;86:154-8.

11. Nissen SE, Yock P. Intravascular ultrasound: novel pathophysiological insights and current clinical applications. Circulation 2001;103:604-16.

12. Glagov $S$, Weisenberg E, Zarins $C$, et al. Compensatory enlargement of human coronary arteries. N Engl J Med 1987;316:1371-5.

13. GerberTC, Erbel R, Gorge G, Ge J, Meyer J. Extent of atherosclerosis and remodeling of the left main coronary artery determined by intravascular ultrasound. Am J Cardiol 1994;73:666-71.

14. Mintz GS, painter JA, Pichard AD, et al. Atherosclerosis in angiographically "normal"coronary artery reference segments: an intravascular ultrasound study with clinical correlations. J Am Coll Cardiol 1995;25:1479-85.

15. Mintz GS, Kent KM, Pichard AD, et al. Contribution of inadequate arterial remodeling to the development of focal coronary artery stenoses: an intravascular ultrasound study. Circulation 1997;95:1791-8.

16. Ambrose J, Winters S, Arora R, et al. Angiographic evolution of coronary artery morphology in unstable angina. J Am Coll Cardiol 1986;7:472-8.

17. Moise A, Theroux $P$, Yaeymans $Y$, et al. Unstable angina and progression of coronary artery disease. N Engl J Med 1983;309:685-9.

18. Richardson PD, Davies MJ, Born GVR. Influence of plaque configuration and stress distribution on fissuring of coronary atherosclerotic plaques. Lancet 1989;21:941-4

19. Ambrose JA, Tannembaum MA, Alexopoulos D, et al. Angiographic progression of coronary artery disease and the development of myocardial infarction. J Am Coll Cardiol 1988;12:56-62.

20. Gorlin R, Fuster V, Ambrose J. Anatomic-physiologic links between acute coronary syndromes. Circulation 1986;74:6-9.

21. Hermiller JB, Tenaglia AN, Kisslo KB, et al. In vivo validation of compensatory enlargement of atherosclerotic coronary arteries. Am J Cardiol.1993;71:665-8.

22. Abizaid AS, Mintz GS, Abizaid A, et al. Onw-Year Follow-up After Intravascular Ultrasound Assessment of Moderate Left Main Coronary Artery Disease in Patients With Ambiguous Angiograms. J Am Coll Cardiol 1999;34:707-15.

23. Abizaid AS, Mintz GS, Mehran R et al. Long-term follow-up after percutaneous transluminal coronary angioplasty was not performed based on intravascular ultrasound findings: importance of lumen dimensions. Circulation 1999; 100:256-61.

24. Mann JM, Davies MJ. Vulnerable plaque: relation of characteristics to degree of stenosis in human coronary arteries. Circulation 1996;94:928-31.

25. Ambrose J, Winters S, Stern A, et al. Angiographic morphology and the pathogenesis of unstable angina pectoris. J Am Coll Cardiol 1985;5:609-14.

26. Falk E, Shah PK, Fuster V. Coronary plaque disruption. Circulation 1995;92: 657-71.

27. Kalbfleisch SJ, McGillem MJ, Simon SB, et al. Automated quantitation of index of coronary lesion complexity. Comparison between patients with stable and unstable angina. Circulation 1990;82:439-47.

28. Kragel AH, Reddy SG, Witters AT, Roberts WC. Morphometric analysis of the composition of coronary arterial plaques in isolated unstable angina pectoris with pain at rest. Am J Cardiol 1990;66:562-7.
29. Kragel AH, Reddy SG, Witters AT, Roberts WC. Morphometric analysis of the composition of atherosclerotic plaques in the four epicardial coronary arteries in acute myocardial infarction and in sudden cardiac death. Circulation 1989;80: 1747-56.

30. Depré C, Wijns W, Robert AM, Renkin JP, Havaux W. pathology of unstable plaque: correlation with the clinical severity of acute coronary syndromes. J Am Coll Cardiol 1997;30:694-702.

31. Gussenhoven Ej, Essed CE, Lancee CT, et al. Arterial wall characteristics determined by intravascular ultrasound imaging: an in vitro study. J Am Coll Cardiol 1989;14:947-52.

32. Kimura BJ, Bhargava V, De Maria NA. Value and limitations of intravascular ultrasound imaging in characterizing coronary atherosclerotic plaque. Am Heart J 1995;130:386-96.

33. Bartorelli AL, Potkin BN, Almagor Y, Keren G, Roberts WC, Leon MB. Plaque characterization of atherosclerotic coronary arteries by intravascular ultrasound. Echocardiography 1990;7:389-95.

34. Waller BF, McKay C, Gessert J, Pinkerton G, Zalesky P. Intravascular ultrasound - a useful technique for mapping plaque topography, recognizing eccentric lumen, arterial dissection and showing the results of coronary angioplasty and atherectomy. J Am Coll Cardiol 1990;15:17A.

35. Loree HM, Kamm RD, Stringfellow RG, Lee RT. Effects of fibrous cap thickness on peak circunferential stress in model atherosclerotic vessels. Circ Res 1992; 71:850-8.

36. Lee RT, Loree HM, Cheng GC, et al. Computational structural analysis based on intravascular ultrasound imaging before in vitro angioplasty: prediction of plaque fracture locations. J Am Coll Cardiol 1993;21:777-82.

37. Chemarin-Alibelli MJ, Pieraggi MT, Elbaz M, et al. Identification of coronary thrombus after myocardial infarction by intracoronary ultrasound compared with histology of tissues sampled by atherectomy. Am J Cardiol 1996;77:344-9.

38. Frimerman A, Miller HI, Hallman M, Laniado S, keren G. Intravascular ultrasound characterization of thrombi of different composition. Am J Cardiol 1994;73:1053-7.

39. Pandian NG, Kreis A, Brockway B. Detection of intra-arterial thrombus by intravascular high frequency two-dimensional ultrasound imaging in in-vitro and in-vivo studies. Am J Cardiol 1990;65:1280-3.

40. Jain A, Ramee SR, Mesa J, Collins TJ, White CJ. Intracoronary thrombus: Chronic urokinase infusion and evaluation with intravascular ultrasound. Cath Cardiovasc Diag 1992;26:212-4.

41. Ge J, Liu F, Goerge G, et al. Visualization of unstable plaque rupture by intracoronary ultrasound. Circulation 1995;1995;196A

42. Hodgson JMcB, Reddy KG, Suneja R, et al. Intracoronary ultrasound imaging: correlation of plaque morphology with angiography, clinical syndrome and procedural results in patients undergoing coronary angioplasty. J Am Coll Cardiol 1993;21:35-44.

43. Rasheed Q, Nair RN, Sheehan HM, Hodgson JM. Coronary artery plaque morphology instable angina and subsets of unstable angina: an in vivo intracoronary ultrasound study. Intern J of Card Imag 1995;11:89-95.

44. Kearney P, Erbel R, Rupprecht HJ, et al. Differences in the morphology of unstable and stable coronary lesions and their impact on the mechanisms of angioplasty: an in vivo study with intravascular ultrasound. Eur Heart J 1996;17:721-30.

45. Bocksch WG, Schartl M, Beckmann SH, et al. intravascular ultrasound imaging in patients with acute myocardial infarction: comparison with chronic stable angina pectoris. Coron Artery Dis 1994;5:727-35.

46. Bocksch W, Schartl M, Beckmann S, et al. intravascular ultrasound assessment of direct percutaneous transluminal coronary angioplasty in patients with acute myocardial infarction. Coron Artery Dis 1997;8:265-73.

47. Birnbaum Y, Luo H, Fishbein MC, et al. Documentation by intravascular ultrasound of thrombus overlying a small atheromatous plaque in a coronary artery in acute myocardial infarction. Am J Cardiol 1997;79:1568-70.

48. Lee TM, Chu CC, Hsu YM, et al. Exaggerated luminal loss a few minutes after successful percutaneous transluminal coronary angioplasty in patients with recent myocardial infarction compared with stable angina: an intravascular ultrasound study. Cath and Cardiovasc Diag 1997;41:32-9.

49. Abizaid AS, Mintz GS, Satler LF, et al. Intravascular ultrasound almost always detects unstable lesion morphology within 72 hours of onset of acute myocardial infarction. J Am Coll Cardiol 1999;33:53A.

50. Feld S, Ganim M, Carell ES, et al. Comparison of angioscopy, intravascular ultrasound imaging and quantitative coronary angiography in predicting clinical outcome after coronary intervention in high risk patients. J Am Coll Cardiol 1996;28:97-105.

51. De Jaegere P, Mudra H, Figulla $\mathrm{H}$, et al. Intravascular ultrasound-guided optimized stent deployment:immediate and 6 months clinical and angiographic 
results from the Multicenter Ultrasound Stenting in Coronaries Study (MUSIC Study). Eur Heart J. 1998;19:1214-23.

52. Goldberg SL, Colombo A, Nakamura S, et al. The benefit of intracoronary ultrasound in the deployment of Palmaz-Schatz stent. J Am Coll Cardiol. 1994;24: 996-1003.

53. Abizaid A. Contribuição do ultra-som intracoronariano como método guia para o implante ótimo do stent de Palmaz-Schatz em 1196 pacientes consecutivos: impacto na restenose clínica. São Paulo, 2000. Tese - doutorado - Faculdade de Medicina da Universidade de São Paulo.

54. Fitzgerald PJ, Oshima A, Hayase M, et al. Final Results of the Can Routine Ultrasound Influence Stent Expansion (CRUISE) study. Circulation. 2000;102:523-30.

55. Mueller C, Mc Hodgson JB, Brutsche M, et al. Impact of intracoronary ultrasound guidance on long-term outcome of percutaneous coronary interventions in diabetics insights from the randomized SIPS trial. Swiss Med Wkly. 2002; 132:21-2.

56. Mudra $\mathrm{H}$, Macaya $\mathrm{C}$, Zahn R, et al. Interim analysis of the "OPTimization with IVUS to reduce stent restenosis" (OPTICUS) trial. Circulation.1998;98:363A.

57. Oemrawsingh PV, Mintz GS, Schalij MJ, et al. Intravascular ultrasound guidance improves angiographic and clinical outcome of stent implantation for long coronary artery stenoses: final results of a randomized comparison with angiographic guidance (TULIP Study). Circulation. 2003; 107: 162-7.

58. Mintz GS, Hoffmann R, Mehran R, et al. In-stent restenosis: the Washington Hospital Center experience. Am J Cardiol 1998;81:7E-13E.
59. Castagha MT, Mintz GS, Leiboff BO, et al. The contribution of "mechanical problems to in-stent restenosis: An intravascular ultrasonographic analysis of 1090 consecutive in-stent restenosis lesions. Am Heart J 2001;142:971-10.

60. Sousa JMR., Costa MA, Abizaid AAC, et al. Lack of neointimal proliferation after implantation of sirolimus-coated stents in human coronary arteries: A QCA and three-dimensional IVUS study. Circulation; 2001,103:192-5.

61. Serruys PW, Degertekin M, Tanabe K, et al. Intravascular ultrasound findings in the multicenter, randomized, double-blind RAVEL (RAndomized study with the sirolimus-eluting VElocity balloon-expandable stent in the treatment of patients with de novo native coronary artery Lesions) trial. Circulation. 2002; 106:798-803.

62. Sousa JE, Costa MA, Abizaid A, et al. Sirolimus-eluting stent for the treatment of in-stent restenosis: a quantitative coronary angiography and three-dimensional intravascular ultrasound study. Circulation. 2003;107:24-7.

63. Sousa JE, Costa MC, Sousa AGMR, et al. Two-year angiographic and intravascular ultrasound follow-up after implantation of sirolimus-eluting stents in human coronary arteries. Circulation. 2003;107:381-3.

64. Tanabe K, Serruys PW, Grube E, et al. TAXUS III Trial: in-stent restenosis treated with stent-based delivery of paclitaxel incorporated in a slow-release polymer formulation. Circulation.2003;107:559-64.

65. Grube E, Silber S, Hauptmann KE, Mueller R et al. TAXUS I: six- anda twelvemonth results froma a randomized, double-blind trial on a slow-release paclitaxeleluting stent for de novo coronary lesions. Circulation. 2003;107:38-42. 